\title{
New Mild and Simple Approach to Isothiocyanates: A Class of Potent Anticancer Agents
}

\author{
Bingling Luo ${ }^{1,2,+}$, Jiankang Wang ${ }^{1,2,+}$, Xiaobing $\mathrm{Li}^{1}{ }^{1}$, Wenhua Lu ${ }^{1}$, Jing Yang ${ }^{1}$, Yumin $\mathrm{Hu}{ }^{1}$, \\ Peng Huang ${ }^{1,2}$ and Shijun Wen ${ }^{1,2, *}$ \\ 1 Sun Yat-Sen University Cancer Center; State Key Laboratory of Oncology in South China; \\ Collaborative Innovation Center for Cancer Medicine; Sun Yat-Sen University, 651 Dongfeng East Road, \\ Guangzhou 510060, China; luobl@sysucc.org.cn (B.L.); wangjk6@mail2.sysu.edu.cn (J.W.); \\ lixiaobing0629@126.com (X.L.); luwenh@sysucc.org.cn (W.L.); yangjing@sysucc.org.cn (J.Y.); \\ huym@sysucc.org.cn (Y.H.); huangpeng@sysucc.org.cn (P.H.) \\ 2 School of Pharmaceutical Sciences, Sun Yat-sen University, 132 Waihuan East Road, \\ Guangzhou 510006, China \\ * Correspondence: wenshj@sysucc.org.cn; Tel.: +86-20-3994-3091 \\ + The authors contributed equally to this work.
}

Academic Editors: Grzegorz Wegrzyn, Luciano Saso, László Dux and Tamás Csont Received: 18 March 2017; Accepted: 27 April 2017; Published: 1 June 2017

\begin{abstract}
In our current work, acetyl chloride-mediated synthesis of phenethyl isothiocyanate (PEITC) derivatives proves to be convenient and provides the expected products at good to excellent yields. Biological evaluation and structure-activity relationship analysis found that the novel compound 7 showed the best anticancer activity against human cancer cell line Panc1 and HGC27 compared with PEITC. Compounds 6 and 7 induced more apoptosis in pancreatic cancer cells but less toxicity in non-cancer cells. Further biological study demonstrated that 7 substantially increased intracellular reactive oxygen species (ROS) and depleted glutathione (GSH), leading to an oxidative stress to kill cancer cell.
\end{abstract}

Keywords: isothiocyanate; anticancer; reactive oxygen species; glutathione

\section{Introduction}

Isothiocyanates are important natural compounds found in cruciferous vegetables such as watercress and broccoli, and they have shown cancer prevention effects [1,2]. Meanwhile, it is also reported that these natural isothiocyanates can induce apoptosis of cancer cells, although its mechanism is still under debate [3-6]. Among these reported natural compounds, one of the most studied is phenethyl isothiocyanate (PEITC). Packham's group found that PEITC could target mTORC1 activity in leukemia cells $[7,8]$, and Chung and his co-workers reported that isothiocyanates selectively depleted mutant p53, compared with wild type p53 [9]. In our previous study, PEITC showed a great selectivity in killing oncogenically transformed cells over normal cells through a reactive oxygen species (ROS)-mediated mechanism [10-12], indicating that PEITC could hold therapeutic promise for the treatment of cancer patients. Indeed, PEITC has been registered for clinical trials for cancer prevention and treatment (ClinicalTrials.gov Identifiers: NCT00005883, NCT00691132, NCT01790204). Due to the potential cancer preventive and therapeutic benefits of PEITC and other isothiocyanates, both biological evaluation and chemical synthesis of these unique compounds have attracted more interest $[9,13]$.

Apart from their potential anticancer effect, isothiocyanates are also valuable intermediates to obtain thioureas. The unique pharmacological properties and structural features of isothiocyanates attract the interest of chemists, and a number of their synthetic routes have been developed. 
The reported methods include using toxic thiophosgene [14], or less toxic but expensive thiophosgene surrogate reagents such as 1,1'-thiocarbonyldiimidazole [15], to incorporate one carbon atom and one sulfur atom. The alternative incorporation of these two atoms employs carbon disulfide through the decomposition of the in situ-formed dithiocarbamate salt intermediates [16-18]. In this strategy, several reagents have been reported, for example, dicyclohexylcarbodiimide [16], 2-chloro-1,3-dimethylimidazo-liniumchloride [17], and the more recently reported di-tert-butyl dicarbonate [18] and tosyl chloride [19]. It is of importance to develop novel and potent isothiocyanates via the optimization of PEITC, while few analogues of PEITC have shown outstanding anticancer activities regarding their in vitro $\mathrm{IC}_{50}$ values. In this paper, we report a concise method to prepare isothiocyanates and conduct a biological evaluation of these compounds. Finally, we find that compound 7 showed the best anti-proliferative activity via modulating the increase of intracellular ROS levels.

\section{Results and Discussion}

\subsection{Chemistry}

During our initial study, the preparation of polar isothiocyanates using tosyl chloride did not encounter any problems. However, in the synthesis of less polar isothiocyanates, a slight excess of tosyl chloride was always difficult to remove from the products by flash chromatography. This problem hampered our progress in obtaining a variety of compounds, thus it is of high interest to find an alternative concise method of doing so. To our best knowledge, there is not yet a generally conceptualized mechanism for the decomposition of dithiocarbamic acid salt mediated by the previously reported reagents. Clearly, the in situ formed dithiocarbamate salt is a potential nucleophile which could react with a nucleophilic receptor, i.e., an electrophile, to form an intermediate A (Scheme 1). Mediated by bases such as triethylamine, an isothiocyanate can be formed after one molecular hydrogen sulfide is eliminated from A and transported to the electrophile. Thus, we envisioned that some electrophiles might be able to mediate the production of isothiocyanates (Scheme 1).

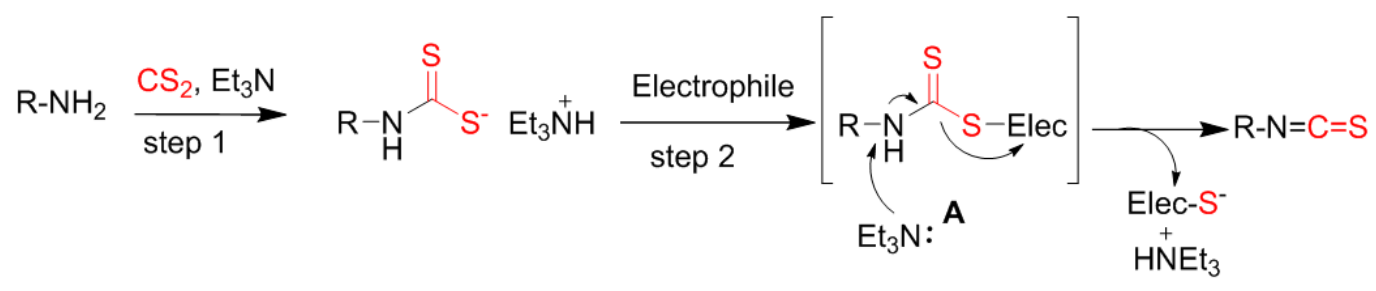

Scheme 1. The potential mechanism for the synthesis of isothiocyanates.

To validate our hypothesis that other electrophiles can replace tosyl chloride, a series of conventional reagents including acetyl chloride $(\mathrm{AcCl})$, tosyl chloride $(\mathrm{TsCl})$, dimethyldichlorosilane $\left(\mathrm{SiMe}_{2} \mathrm{Cl}_{2}\right)$, benzoyl chloride $(\mathrm{BzCl})$ and sulfonyl chloride $\left(\mathrm{SO}_{2} \mathrm{Cl}_{2}\right)$ were screened for the synthesis of PEITC from phenethylamine (Table 1). Indeed, all reagents resulted in the expected product in modest to good yields, ensuring that the decomposition of the dithiocarbmate was mediated by its nucleophilic attack on an oncoming electrophile in step 2 (Scheme 1). While the excess tosyl chloride cannot be cleared after the reaction work-up, causing an issue with the purification of low polar isothiocyanates by flash chromatography, acetyl chloride could avoid such a problem and also provided excellent yields. Thus, acetyl chloride was employed to prepare various isothiocyanates in this current work. It is noteworthy that analytic grade tetrahydrofuran (THF) and triethylamine might be used in the late synthesis of some isothiocyanates although the reactions were performed under anhydrous conditions in the screening study. It was also found that triethylamine could be replaced by inorganic bases such as potassium carbonate (data not shown). 
Table 1. Synthesis of phenethyl isothiocyanate mediated by various reagents.

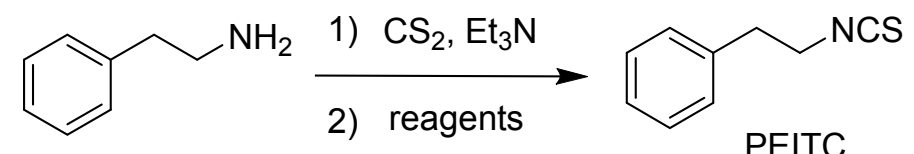

\begin{tabular}{cccccc}
\hline Entry & $\mathbf{1}$ & $\mathbf{2}$ & $\mathbf{3}$ & $\mathbf{4}$ & $\mathbf{5}$ \\
\hline Reagent & $\mathrm{AcCl}$ & $\mathrm{TsCl}$ & $\mathrm{SiMe}_{2} \mathrm{Cl}_{2}$ & $\mathrm{BzCl}$ & $\mathrm{SO}_{2} \mathrm{Cl}_{2}$ \\
Yield & $94 \%$ & $70 \%$ & $79 \%$ & $42 \%$ & $80 \%$
\end{tabular}

Reagents and Conditions: (1) phenylethylamine (1 equiv.), $\mathrm{CS}_{2}$ (1.2 equiv.), and $\mathrm{Et}_{3} \mathrm{~N}$ (3 equiv.) in anhydrous THF, $0{ }^{\circ} \mathrm{C}$-r.t.; (2) reagents (1.2 equiv.) was added, $0^{\circ} \mathrm{C}-$ r.t.

During our study, the preparation of isothiocyanates $\mathbf{1}$ and $\mathbf{2}$ actually did not encounter any problems when tosyl chloride was applied. However, the purification of the low polar isothiocyanates 3-5 encountered difficulty in the full removal of excess tosyl chloride from the compounds. This problem was successfully avoided when acetyl chloride was employed (Figure 1). The remaining isothiocyanates 6-20 were also easily prepared at excellent yields, similar to or better than the yields obtained with tosyl chloride used, as exemplified in the synthesis of 4,6 and 7 .

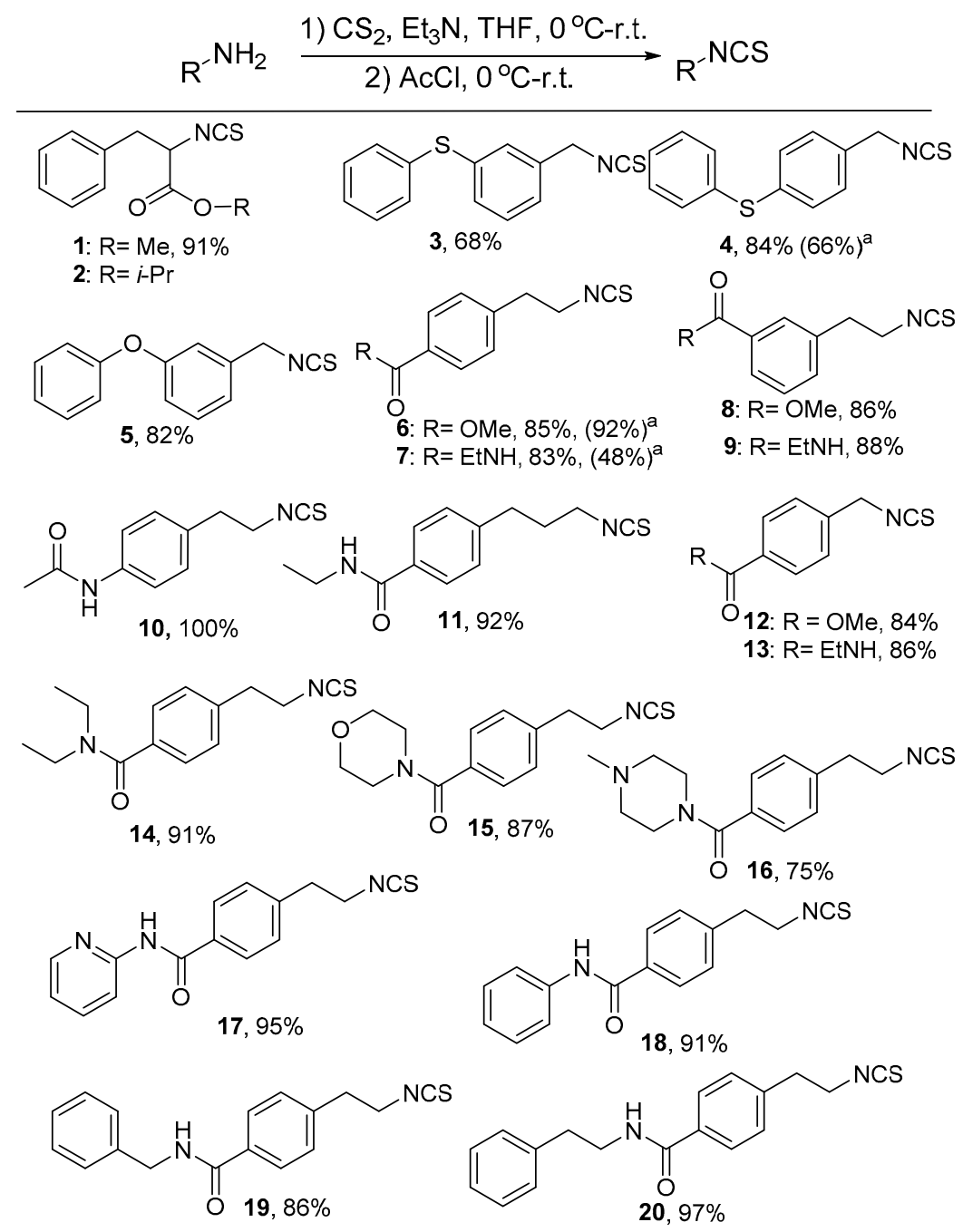

Figure 1. Structures of isothiocyanates and their preparation using acetyl chloride. Note: Unless otherwise stated, all the yields are isolated yields. ${ }^{a}$ The number in the brackets indicates the yield when prepared with tosyl chloride. 


\subsection{Biology}

To design isothiocyanates with better bioactivity is a challenge, because the mechanism of their inhibition of tumor cell growth is still debated and their exact targets remain unclear. Based on our experience, we envisioned that aromatic rings and a chain with reasonable length might be essential to maintain bioactivity. The substituents on the aromatic ring, varying in space size and chemical properties, might influence the compound's selectivity to bind to certain targets. Thus, isothiocyanates $\mathbf{1}$ and $\mathbf{2}$ (Figure 1) were firstly designed and they were prepared from a phenylalanine. Then, 3-5 were obtained, identified by another benzene ring tethered through an oxygen or sulfur atom on either the meta- or para-position of PEITC. Such structural variation would justify whether a bulky group could increase its selectivity or binding affinity to certain targets. Furthermore, compounds 6 and 7 were designed and synthesized because of the possibility that such functional amides and esters might result in an improvement of PEITC bioavailability and the formation of potential hydrogen bonds with some potential targets.

In our preliminary study, isothiocyanates 1-7 were screened against two types of malignant cancer cell lines, Panc 1 (pancreatic cancer) and HGC 27 (gastric cancer), since these pancreatic and gastric cancers are fatal and have a poor prognosis (Table 2) [20-23].

Table 2. Anti-proliferative activities of isothiocyanates against two types of human cancer cell lines, Panc1 (pancreatic cancer) and HGC27 (gastric cancer), measured by MTS assays.

\begin{tabular}{ccc}
\hline \multirow{2}{*}{ Compound } & \multicolumn{2}{c}{ IC $_{\mathbf{5 0}}(\boldsymbol{\mu M})$} \\
\cline { 2 - 3 } & Panc1 & HGC27 \\
\hline PEITC & $10.80 \pm 0.25$ & $2.95 \pm 0.41$ \\
$\mathbf{1}$ & $15.80 \pm 1.06$ & $8.79 \pm 0.72$ \\
$\mathbf{2}$ & $10.87 \pm 0.47$ & $1.49 \pm 0.06$ \\
$\mathbf{3}$ & $14.24 \pm 0.50$ & $2.73 \pm 0.29$ \\
$\mathbf{4}$ & $6.12 \pm 0.13$ & $3.44 \pm 0.23$ \\
$\mathbf{5}$ & $8.05 \pm 0.36$ & $2.67 \pm 0.37$ \\
$\mathbf{6}$ & $4.55 \pm 0.32$ & $1.09 \pm 0.07$ \\
$\mathbf{7}$ & $2.04 \pm 0.21$ & $0.46 \pm 0.02$ \\
$\mathbf{8}$ & $5.78 \pm 0.56$ & $0.74 \pm 0.01$ \\
$\mathbf{9}$ & $3.52 \pm 0.11$ & $0.84 \pm 0.06$ \\
$\mathbf{1 0}$ & $2.68 \pm 0.21$ & $0.93 \pm 0.41$ \\
$\mathbf{1 1}$ & $5.08 \pm 0.02$ & $1.47 \pm 0.10$ \\
$\mathbf{1 2}$ & $8.92 \pm 0.43$ & $0.72 \pm 0.21$ \\
$\mathbf{1 3}$ & $5.65 \pm 0.16$ & $0.99 \pm 0.15$ \\
$\mathbf{1 4}$ & $2.25 \pm 0.21$ & $0.62 \pm 0.04$ \\
$\mathbf{1 5}$ & $3.76 \pm 0.05$ & $1.68 \pm 0.00$ \\
$\mathbf{1 6}$ & $6.24 \pm 0.13$ & $2.37 \pm 0.43$ \\
$\mathbf{1 7}$ & $4.70 \pm 0.27$ & $0.77 \pm 0.18$ \\
$\mathbf{1 8}$ & $2.78 \pm 0.72$ & $0.34 \pm 0.00$ \\
$\mathbf{1 9}$ & $2.14 \pm 0.79$ & $0.72 \pm 0.08$ \\
$\mathbf{2 0}$ & $4.06 \pm 0.57$ & $0.82 \pm 0.06$ \\
\hline
\end{tabular}

Compared to PEITC, the new synthetic isothiocyanates $\mathbf{1}$ and $\mathbf{2}$ showed worse or similar bioactivity especially against Panc 1 , implying that extra carboxylate function groups next to the isothiocyanate do not contribute to the biological activity of PEITC. In isothiocyanates 3-5, additional bulky benzene ring did not increase their anticancer activity much compared to PEITC. However, the carboxylate functional groups in isothiocyanates $\mathbf{6}$ and $\mathbf{7}$ substantially improved their ability to kill cancer cells. In particular, compound 7 with the para amide on the benzene ring of PEITC showed the best potency with 5 or 6 fold increasement. Based on the structure of 7, the subsequent isothiocyanates 8-20 were designed and prepared in order to study their structure and activity relationship. Among the designed compounds, the carboxylate functional groups remained unchanged because they might contribute to 
the increment of inhibitory effects, likely by either increasing bioavailability or by forming hydrogen bonds with potential targeted macromolecules.

Compounds $\mathbf{8}$ and $\mathbf{9}$ with respective meta-positioned ester and amide functional groups showed a slightly lower anticancer activity compared to 6 and 7, implying that substituents on the para-position was better. Compound 10, having an acetamide replace the ethyl amide in 7, also showed similar potency to 7 , further confirming the importance of the carboxylate function groups in the para-position. The length variation of chains linking the benzene ring and isothiocyanate function group provided compounds 11-13. The subsequent biological evaluation demonstrated that a longer or shorter chain of $\mathbf{7}$ diminished its potency, while a two-carbon linker provided the best potency. This finding implied that the chain length might be crucial for maintaining a balance between rigidity and flexibility to gain more potency. Thus, more structural modifications were performed on the skeleton of 7 ; varying the amine motif provided compounds 14-20, with the ethyl amine in 7 replaced by diethyl amine, morpholine, piperazine, pyridine-2-amine, aniline, and other amines (Figure 1). The variation of the amine motif in 7 did not gain more potency to inhibit cancer growth in the newly prepared compounds 14-20. Thus, compound 7 was selected for further biological study, using PEITC and 6 for comparison.

Pancreatic cancer is one of the most fatal human cancers which remains a challenging health problem due to its high metastases [24]. Our further biological evaluation of compound 7 focused on the human pancreatic cancer cell lines Panc1 and Capan2 (Figure 2). Compound 7 showed better anti-proliferative capability in a dose-dependent manner compared to PEITC and 6, as shown in Figure 2a. In the colony formation experiments, 7 substantially inhibited Panc1 growth even at $1 \mu \mathrm{M}$ concentration (Figure $2 \mathrm{~b}$ ). Compared to PEITC, $\mathbf{6}$ and $\mathbf{7}$ induced higher apoptosis of Panc1 and Capan2 after $72 \mathrm{~h}$ treatment at $10 \mu \mathrm{M}$ concentration (Figure 3a). However, 6 and 7 caused similar or less cytotoxicity to non-cancerous cells, as evidenced by the cell viability of E6E7 that is an immortalized human pancreatic ductal epithelium cell line (Figure 3b).

(a)

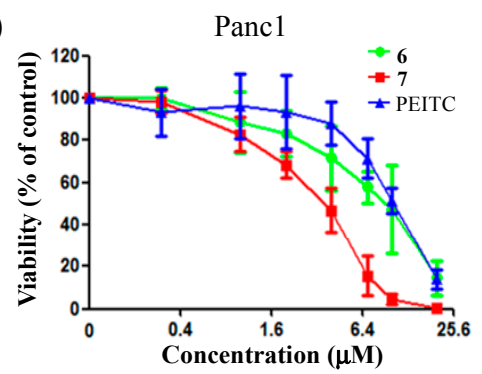

(b)

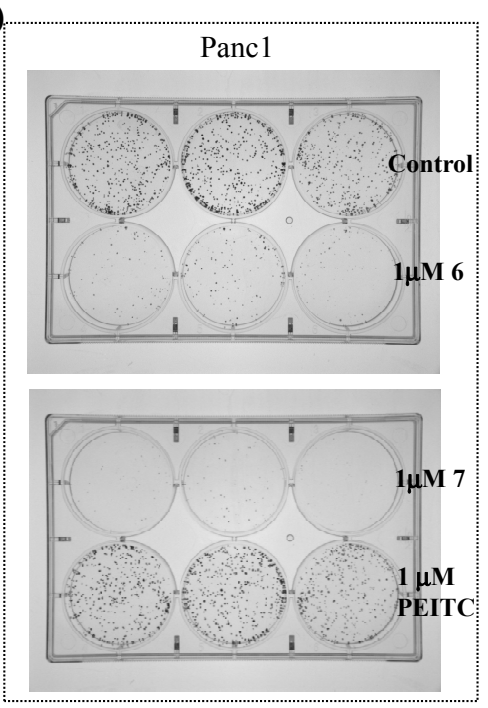

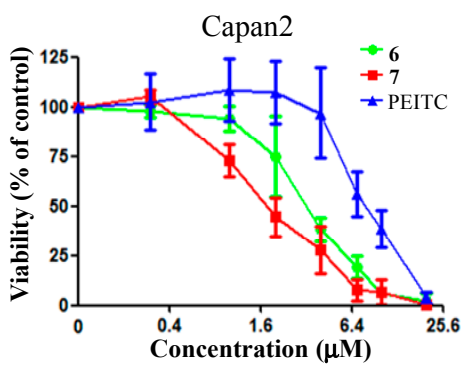

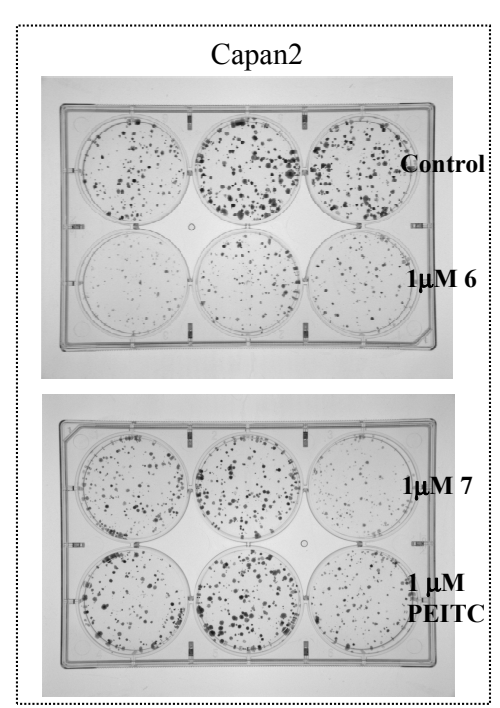

Figure 2. The cellular toxicity of $\mathbf{6}$ and $\mathbf{7}$ on the human pancreatic cancer cell lines Panc1 and Capan2. (a) MTS assay after $72 \mathrm{~h}$ treatment; (b) 14-day colony formation (triplicate wells). 


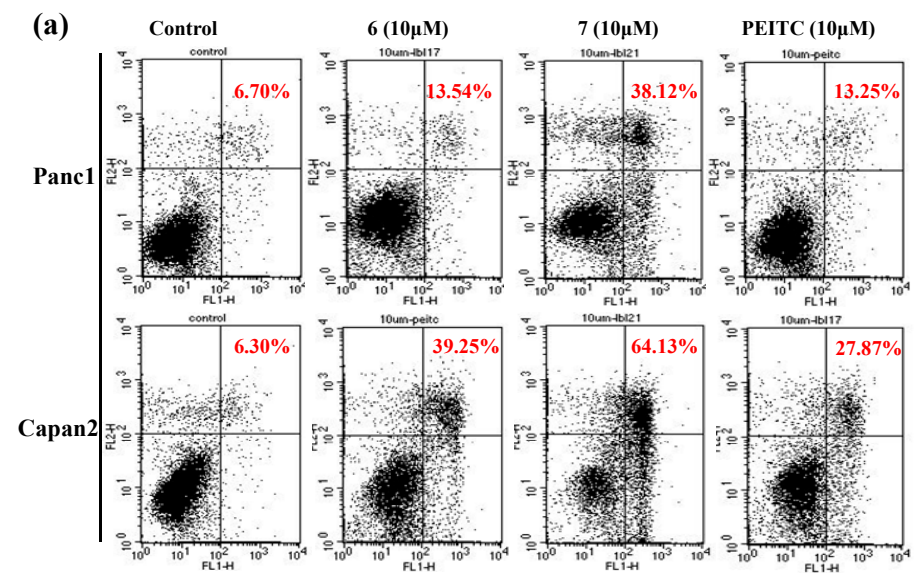

(b)

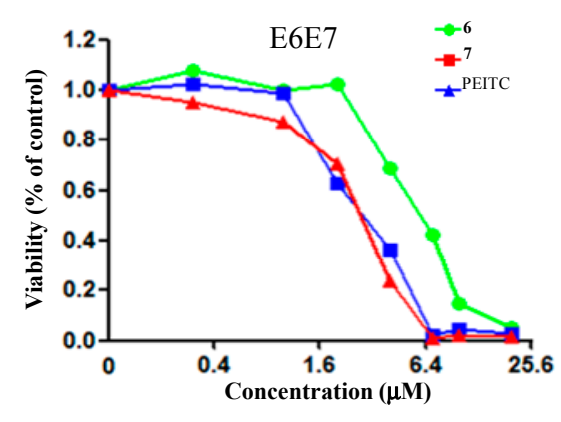

Figure 3. (a) The apoptosis induction caused by 6 and 7 in pancreatic cancer cells. Percentage numbers show annexin-V/PI positive cell fraction; (b) E6E7 cells were treated with various agents for $72 \mathrm{~h}$ before MTS assay.

ROS play important roles in the regulation of cell survival and cell death. In general, a moderate ROS level promotes cell proliferation, whereas an excessive increase of ROS can induce cell death by causing cellular redox imbalance $[25,26]$. Since PEITC killed malignant cancer cells via the ROS modulation pathway, the effect of 6 and 7 on intracellular ROS levels was finally tested. Indeed, 7 significantly increased the ROS level as PEITC, as shown in Figure 4a. Intracellular glutathione (GSH) is a very important endogenous antioxidant to defend high oxidative stress in malignant cancer cells $[27,28]$. Thus, we also tested whether our synthetic isothiocyanates 6 and 7 could deplete GSH in Panc1 and Capan 2 cells (Figure 4b,c). As expected, GSH depletion by 7 was the most significant, followed by 6 and PEITC, consistent to their anticancer activity.

(a)

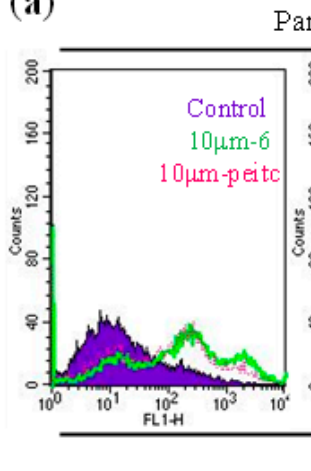

Panc1

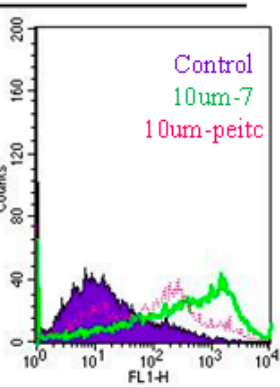

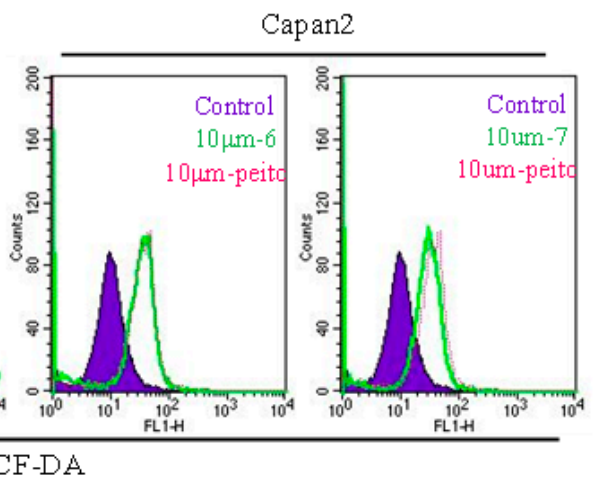

(b)

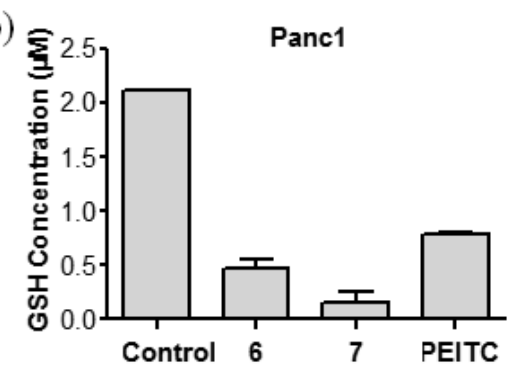

(c)

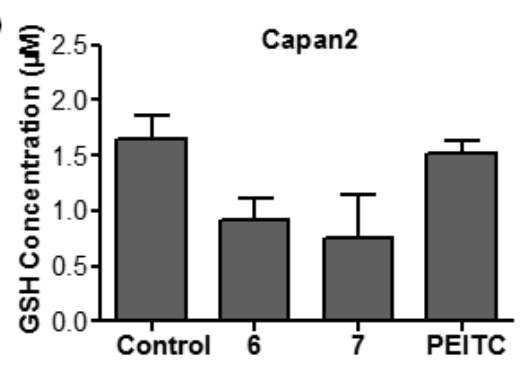

Figure 4. Compounds 6 and 7 induced a redox imbalance in pancreatic cancer cells. (a) The content of hydrogen peroxide (6 h); (b,c) the content of cellular GSH in Panc1 (b) and Capan2 (c) treated for $2 \mathrm{~h}$ with compounds at a concentration of $10 \mu \mathrm{M}$. 


\section{Materials and Methods}

\subsection{General Procedure Exemplified by the Synthesis of PEITC}

All reactions were performed under argon, unless indicated otherwise. Column chromatography was performed using silca gel (200-300 mesh). NMR spectra were recorded on a Bruker $400 \mathrm{MHz}$ spectrometer (Rheinstetten, Germany). ${ }^{1} \mathrm{H}-\mathrm{NMR}$ spectra were referenced internally to either $\mathrm{CDCl}_{3}$ $(\delta 7.26 \mathrm{ppm})$ or DMSO- $d_{6}(\delta 2.50 \mathrm{ppm}) .{ }^{13} \mathrm{C}-\mathrm{NMR}$ spectra were referenced to $\mathrm{CDCl}_{3}(\delta 77.16 \mathrm{ppm}$, the middle peak) or DMSO- $d_{6}(\delta 39.52 \mathrm{ppm}$, the middle peak). Chemical shifts $(\delta)$ were reported as part per million (ppm), and coupling constants $(J)$ reported in Hertz $(\mathrm{Hz})$. Mass spectra were recorded with an Agilent LC-MS 6120 spectrometer (Santa Clara, CA, USA). High-resolution mass spectrometry (HRMS) was carried out using a Shimadzu LCMS-IT-TOF spectrometer (Kyoto, Japan). ${ }^{1} \mathrm{H}-\mathrm{NMR}$ spectrum and ${ }^{13} \mathrm{C}-\mathrm{NMR}$ spectrum can be found at Supplementary Materials.

To a solution of phenethylamine $(200 \mathrm{mg}, 1.65 \mathrm{mmol})$ and $\mathrm{Et}_{3} \mathrm{~N}(0.64 \mathrm{~mL}, 4.95 \mathrm{mmol})$ in anhydrous THF $(2.5 \mathrm{~mL})$ cooled with an ice bath, a solution of $\mathrm{CS}_{2}(0.12 \mathrm{~mL}, 1.98 \mathrm{mmol})$ was slowly dropped in. The reaction solution was stirred at room temperature for $0.5 \mathrm{~h}$, after which $\mathrm{AcCl}(0.14 \mathrm{~mL}, 1.98 \mathrm{mmol})$ was dropped in at $0{ }^{\circ} \mathrm{C}$, and after $5 \mathrm{~min}$ the mixture was warmed to room temperature for $15-30 \mathrm{~min}$. When the starting amine was finished, as verified by checking thin layer chromatography (T.L.C.), $1 \mathrm{M} \mathrm{HCl}$ (aq., $2 \mathrm{~mL}$ ) was added to quench the reaction. The solution was extracted by EtOAc three times. All organic phases were combined and washed with brine, dried over $\mathrm{Na}_{2} \mathrm{SO}_{4}$, and finally filtered and concentrated under reduced pressure. The residue was purified by column chromatography on silica gel $($ EtOAc/petroleum $=1 / 1)$ to provide phenethyl isothiocyanate (PEITC) $(253 \mathrm{mg}, 94 \%)$.

\subsection{Characterization of Synthetic Isothiocyanates}

Phenethyl isothiocyanate/PEITC. Yellow liquid, yield: $94 \%,{ }^{1} \mathrm{H}-\mathrm{NMR}\left(400 \mathrm{~Hz}, \mathrm{CDCl}_{3}\right): \delta 7.25-7.40$ $(\mathrm{m}, 5 \mathrm{H}), 3.75(\mathrm{t}, 2 \mathrm{H}, \mathrm{J}=6.8 \mathrm{~Hz}), 3.02(\mathrm{t}, 2 \mathrm{H}, J=6.8 \mathrm{~Hz})$.

Methyl 2-isothiocyanato-3-phenylpropanoate (1). Pale yellow liquid, yield: 91\%, ${ }^{1} \mathrm{H}-\mathrm{NMR}\left(400 \mathrm{~Hz} \mathrm{CDCl}_{3}\right)$ : $\delta 7.30-7.38(\mathrm{~m}, 3 \mathrm{H}), 7.21-7.26(\mathrm{~m}, 2 \mathrm{H}), 4.48(\mathrm{dd}, 1 \mathrm{H}, J=4.8,8.0 \mathrm{~Hz}), 3.81(\mathrm{~s}, 3 \mathrm{H}), 3.25(\mathrm{dd}, 1 \mathrm{H}, J=4.8$, $14.0 \mathrm{~Hz}), 3.13(\mathrm{dd}, 1 \mathrm{H}, J=8.0,14.0 \mathrm{~Hz})$.

Isopropyl 2-isothiocyanato-3-phenylpropanoate (2). Yellow liquid, yield: $87 \%,{ }^{1} \mathrm{H}-\mathrm{NMR}\left(400 \mathrm{~Hz}, \mathrm{CDCl}_{3}\right)$ : 反 7.22-7.35 (m, 5H), $5.06(\mathrm{~m}, 1 \mathrm{H}), 4.40(\mathrm{dd}, 1 \mathrm{H}, J=5.2,8.0 \mathrm{~Hz}), 3.22(\mathrm{dd}, 1 \mathrm{H}, J=4.8,13.6 \mathrm{~Hz}), 3.12$ $(\mathrm{dd}, 1 \mathrm{H}, J=8.0,13.6 \mathrm{~Hz}), 1.26(\mathrm{~s}, 3 \mathrm{H}), 1.25(\mathrm{~S}, 3 \mathrm{H}) ;{ }^{13} \mathrm{C}-\mathrm{NMR}\left(100 \mathrm{~Hz}, \mathrm{CDCl}_{3}\right): \delta 167.5,135.3,129.5$, $128.8,127.7,70.8,61.0,39.8,21.8$. HRMS calcd for $\mathrm{C}_{13} \mathrm{H}_{14} \mathrm{NO}_{2} \mathrm{~S}^{-}: 248.0751$, found 248.0747.

(4-(Isothiocyanatomethyl)phenyl)(phenyl)sulfane (3). Pale yellow liquid, yield: $84 \%,{ }^{1} \mathrm{H}-\mathrm{NMR}(400 \mathrm{~Hz}$, $\left.\mathrm{CDCl}_{3}\right): \delta 7.14-7.32(\mathrm{~m}, 9 \mathrm{H}), 4.59$ (s, 2H) ppm; ${ }^{13} \mathrm{C}-\mathrm{NMR}\left(100 \mathrm{~Hz}, \mathrm{CDCl}_{3}\right): \delta 137.2,134.9,133.0132 .0$ $130.9,129.5,127.8,127.7,48.5$ ppm. HRMS calcd for $\mathrm{C}_{14} \mathrm{H}_{10} \mathrm{NS}_{2}{ }^{-}$: 256.0260, found 256.0266.

(3-(Isothiocyanatomethyl)phenyl)(phenyl)sulfane (4). Yellow liquid, yield: $68 \%,{ }^{1} \mathrm{H}-\mathrm{NMR}\left(400 \mathrm{~Hz}, \mathrm{CDCl}_{3}\right)$ : $\delta$ 7.44-7.19 (m, 9H), 4.68 (s, 2H) ppm; ${ }^{13} \mathrm{C}-\mathrm{NMR}\left(100 \mathrm{~Hz}, \mathrm{CDCl}_{3}\right): \delta 137.9,135.5,132.1,130.1,129.8,129.6$, 129.1, 128.4, 127.9, 127.0, 125.2, 48.5 ppm. HRMS calcd for $\mathrm{C}_{14} \mathrm{H}_{10} \mathrm{NS}_{2}{ }^{-}$: 256.0260, found 256.0255.

1-(Isothiocyanatomethyl)-3-phenoxybenzene (5). Pale yellow liquid, yield: $82 \%,{ }^{1} \mathrm{H}-\mathrm{NMR}\left(400 \mathrm{~Hz} \mathrm{CDCl}_{3}\right)$ : $\delta 7.32-7.39(\mathrm{~m}, 3 \mathrm{H}), 7.15(\mathrm{t}, 1 \mathrm{H}, J=7.2 \mathrm{~Hz}), 6.96-7.06(\mathrm{~m}, 5 \mathrm{H}), 4.67(\mathrm{~s}, 2 \mathrm{H}) \mathrm{ppm} ;{ }^{13} \mathrm{C}-\mathrm{NMR}(100 \mathrm{~Hz}$, $\left.\mathrm{CDCl}_{3}\right): \delta 158.1,156.7,136.3,130.5,130.0,123.9,121.5,119.4,118.5,117.1,48.5$ ppm.

Methyl4-(2-isothiocyanatoethyl)benzoate (6). White solid, yield: $85 \%,{ }^{1} \mathrm{H}-\mathrm{NMR}\left(400 \mathrm{~Hz}, \mathrm{CDCl}_{3}\right): \delta 8.01$ $(\mathrm{d}, 2 \mathrm{H}, J=8.0 \mathrm{~Hz}), 7.29(\mathrm{~d}, 2 \mathrm{H}, J=8.0 \mathrm{~Hz}), 3.91(\mathrm{~s}, 3 \mathrm{H}), 3.75(\mathrm{t}, 2 \mathrm{H}, J=6.8 \mathrm{~Hz}), 3.03(\mathrm{t}, 2 \mathrm{H}, J=6.8 \mathrm{~Hz}) \mathrm{ppm}$; ${ }^{13} \mathrm{C}-\mathrm{NMR}\left(100 \mathrm{~Hz}, \mathrm{CDCl}_{3}\right): \delta 166.9,142.4,130.2,129.3,129.0,52.2,46.0,36.5$ ppm; m.p. $119.9-123.1{ }^{\circ} \mathrm{C}$; MS (ESI, $m / z) 222.0\left(\mathrm{M}+\mathrm{H}^{+}\right)$.

N-Ethyl-4-(2-isothiocyanatoethyl)benzamide (7): White solid, yield: 83\%, ${ }^{1} \mathrm{H}-\mathrm{NMR}\left(400 \mathrm{~Hz}, \mathrm{CDCl}_{3}\right): \delta 7.67$ $(\mathrm{d}, 2 \mathrm{H}, J=8.0 \mathrm{~Hz}), 7.21(\mathrm{~d}, 2 \mathrm{H}, J=8.0 \mathrm{~Hz}), 6.05(\mathrm{brs}, 1 \mathrm{H}), 3.68(\mathrm{t}, 2 \mathrm{H}, J=6.4 \mathrm{~Hz}), 3.43(\mathrm{t}, 2 \mathrm{H}, J=6.4 \mathrm{~Hz})$, 
$2.96(\mathrm{t}, 2 \mathrm{H}, J=6.4 \mathrm{~Hz}), 1.18(\mathrm{t}, 3 \mathrm{H}, J=7.2 \mathrm{~Hz}) \mathrm{ppm} ;{ }^{13} \mathrm{C}-\mathrm{NMR}\left(100 \mathrm{~Hz}, \mathrm{CDCl}_{3}\right): \delta 167.1,140.6,134.0$, 129.1, 127.5, 46.2, 36.4, 35.1, 15.0 ppm; m.p. 89.8-92.5 ${ }^{\circ} \mathrm{C}$; HRMS calcd for $\mathrm{C}_{12} \mathrm{H}_{14} \mathrm{~N}_{2} \mathrm{OSNa}^{+}$: 257.0719, found 257.0722 .

Methyl 3-(2-isothiocyanatoethyl)benzoate (8). White solid, yield: $86 \%,{ }^{1} \mathrm{H}-\mathrm{NMR}\left(400 \mathrm{~Hz}, \mathrm{CDCl}_{3}\right)$ : $\delta 7.96-7.94(\mathrm{~m}, 1 \mathrm{H}), 7.90(\mathrm{~s}, 1 \mathrm{H}), 7.42(\mathrm{~d}, 2 \mathrm{H}, J=4.4 \mathrm{~Hz}), 3.92(\mathrm{~s}, 3 \mathrm{H}), 3.76(\mathrm{t}, 2 \mathrm{H}, J=6.8 \mathrm{~Hz}), 3.04(\mathrm{t}, 2 \mathrm{H}$, $J=6.8 \mathrm{~Hz}) ;{ }^{13} \mathrm{C}-\mathrm{NMR}\left(100 \mathrm{~Hz}, \mathrm{CDCl}_{3}\right): \delta 166.9,137.5,133.6,130.8,129.9,129.0,128.6,52.3,46.2,36.3$ ppm; m.p. 86.5-90.8 ${ }^{\circ} \mathrm{C}$; HRMS calcd for $\mathrm{C}_{11} \mathrm{H}_{11} \mathrm{NO}_{2} \mathrm{SNa}^{+}$: 244.0403, found 244.0397.

$\mathrm{N}$-Ethyl-3-(2-isothiocyanatoethyl)benzamide (9). Yellow solid, yield: $88 \%,{ }^{1} \mathrm{H}-\mathrm{NMR}\left(400 \mathrm{~Hz}, \mathrm{CDCl}_{3}\right)$ : $\delta 7.62-7.67(\mathrm{~m}, 1 \mathrm{H}), 7.62(\mathrm{~s}, 1 \mathrm{H}), 7.27-7.32(\mathrm{~m}, 2 \mathrm{H}), 6.51(\mathrm{brs}, 1 \mathrm{H}), 3.65(\mathrm{dd}, 2 \mathrm{H}, J=11.2,6.4 \mathrm{~Hz})$, 3.34-3.52 (m, 2H), $2.93(\mathrm{dd}, 2 \mathrm{H}, J=11.2,6.4 \mathrm{~Hz}), 1.17(\mathrm{t}, 3 \mathrm{H}, J=8.0 \mathrm{~Hz}) ;{ }^{13} \mathrm{C}-\mathrm{NMR}\left(100 \mathrm{~Hz}, \mathrm{CDCl}_{3}\right): \delta$ 167.4, 137.5, 135.6, 131.8, 129.1, 127.6, 126.0, 46.3, 36.4, 35.1, 14.9. HRMS calcd for $\mathrm{C}_{12} \mathrm{H}_{14} \mathrm{~N}_{2} \mathrm{OSNa}^{+}$: 257.0719, found 257.0714.

$\mathrm{N}$-(4-(2-Isothiocyanatoethyl)phenyl)acetamide (10). White solid, yield: $100 \%,{ }^{1} \mathrm{H}-\mathrm{NMR}\left(400 \mathrm{~Hz}, \mathrm{DMSO}-d_{6}\right)$ : $\delta 9.90$ (brs, 1H), $7.52(\mathrm{~d}, 2 \mathrm{H}, J=8.2 \mathrm{~Hz}), 7.19(\mathrm{~d}, 2 \mathrm{H}, J=8.2 \mathrm{~Hz}), 3.86(\mathrm{t}, 2 \mathrm{H}, J=6.4 \mathrm{~Hz}), 2.89(\mathrm{t}, 2 \mathrm{H}$, $J=6.4 \mathrm{~Hz}), 2.03(\mathrm{~s}, 3 \mathrm{H}) \mathrm{ppm} ;{ }^{13} \mathrm{C}-\mathrm{NMR}\left(100 \mathrm{~Hz}, \mathrm{DMSO}-d_{6}\right): \delta 168.1,138.0,132.0,129.0,119.0,46.0$, 34.7, 23.9 ppm; m.p. 154.0-158.1 ${ }^{\circ} \mathrm{C}$; MS (ESI, $\left.m / z\right) 221.1\left(\mathrm{M}+\mathrm{H}^{+}\right), 441.1\left(2 \mathrm{M}+\mathrm{H}^{+}\right)$; HRMS calcd for $\mathrm{C}_{11} \mathrm{H}_{11} \mathrm{~N}_{2} \mathrm{OS}^{-}:$219.0598, found 219.0602.

$\mathrm{N}$-Ethyl-4-(3-isothiocyanatopropyl)benzamide (11). Colorless oil, yield: $92 \%,{ }^{1} \mathrm{H}-\mathrm{NMR}\left(400 \mathrm{~Hz}, \mathrm{CDCl}_{3}\right)$ : $\delta 7.71(\mathrm{~d}, 2 \mathrm{H}, J=8.0 \mathrm{~Hz}), 7.25(\mathrm{~d}, 2 \mathrm{H}, J=8.0 \mathrm{~Hz}), 6.10(\mathrm{brs}, 1 \mathrm{H}), 3.53-3.46(\mathrm{~m}, 4 \mathrm{H}), 2.80(\mathrm{t}, 2 \mathrm{H}, J=7.2 \mathrm{~Hz})$, 1.99-2.06 (m, 2H), $1.25(\mathrm{t}, 3 \mathrm{H}, J=7.2 \mathrm{~Hz}) \mathrm{ppm} ;{ }^{13} \mathrm{C}-\mathrm{NMR}\left(100 \mathrm{~Hz}, \mathrm{CDCl}_{3}\right): \delta 167.4,143.7,133.1,128.7$, 127.4, 44.2, 35.0, 32.5, 31.2, 15.0 ppm. MS (ESI, $m / z) 249.1\left(\mathrm{M}+\mathrm{H}^{+}\right)$, $497.2\left(2 \mathrm{M}+\mathrm{H}^{+}\right)$; HRMS calcd for $\mathrm{C}_{13} \mathrm{H}_{15} \mathrm{~N}_{2} \mathrm{OS}^{-}:$247.0911, found 247.0914.

Methyl 4-(isothiocyanatomethyl)benzoate (12). Brown liquid, yield: $84 \%,{ }^{1} \mathrm{H}-\mathrm{NMR}\left(400 \mathrm{~Hz}, \mathrm{CDCl}_{3}\right): \delta 8.05$ $(\mathrm{d}, 2 \mathrm{H}, J=8.0 \mathrm{~Hz}), 7.38(\mathrm{~d}, 2 \mathrm{H}, J=8.0 \mathrm{~Hz}), 4.77(\mathrm{~s}, 2 \mathrm{H}), 3.91(\mathrm{~s}, 3 \mathrm{H}) \mathrm{ppm} ;{ }^{13} \mathrm{C}-\mathrm{NMR}\left(100 \mathrm{~Hz}, \mathrm{CDCl}_{3}\right)$ : $\delta 166.5,139.3,130.3,126.8,52.33,48.5 \mathrm{ppm}$.

N-Ethyl-4-(isothiocyanatomethyl)benzamide (13). White solid, yield: $86 \%,{ }^{1} \mathrm{H}-\mathrm{NMR}\left(400 \mathrm{~Hz}, \mathrm{CDCl}_{3}\right)$ : $\delta 7.90(\mathrm{~d}, 2 \mathrm{H}, J=8.4 \mathrm{~Hz}), 7.37(\mathrm{~d}, 2 \mathrm{H}, J=8.4 \mathrm{~Hz}), 6.14(\mathrm{brs}, 1 \mathrm{H}), 4.76(\mathrm{~s}, 2 \mathrm{H}), 3.54-3.47(\mathrm{~m}, 2 \mathrm{H}), 1.26$ $(\mathrm{t}, 3 \mathrm{H}, J=7.2 \mathrm{~Hz}) ;{ }^{13} \mathrm{C}-\mathrm{NMR}\left(100 \mathrm{~Hz}, \mathrm{CDCl}_{3}\right): \delta 166.8,137.6,135.0,127.7,127.0,48.5,35.1,15.0 \mathrm{ppm}$; m.p. 94.6-98.7 ${ }^{\circ} \mathrm{C}$; HRMS calcd for $\mathrm{C}_{11} \mathrm{H}_{11} \mathrm{~N}_{2} \mathrm{OS}^{-}$: 219.0598, found 219.0592.

$\mathrm{N}, \mathrm{N}$-Diethyl-4-(2-isothiocyanatoethyl)benzamide (14). Pale brown liquid, yield: $91 \%,{ }^{1} \mathrm{H}-\mathrm{NMR}(400 \mathrm{~Hz}$, $\left.\mathrm{CDCl}_{3}\right): \delta 7.32-7.34(\mathrm{~d}, 2 \mathrm{H}, J=8.0 \mathrm{~Hz}), 7.22-7.24(\mathrm{~d}, 2 \mathrm{H}, J=8.0 \mathrm{~Hz}), 3.72(\mathrm{t}, 2 \mathrm{H}, J=6.8 \mathrm{~Hz}), 3.52(\mathrm{brs}, 2 \mathrm{H})$, $3.24(\mathrm{brs}, 2 \mathrm{H}), 2.99(\mathrm{t}, 2 \mathrm{H}, J=6.8 \mathrm{~Hz}), 1.10-1.24(\mathrm{~m}, 6 \mathrm{H}) \mathrm{ppm} ;{ }^{13} \mathrm{C}-\mathrm{NMR}\left(100 \mathrm{~Hz}, \mathrm{CDCl}_{3}\right): \delta 171.1,138.2$, $136.3,129.0,126.9,46.2,43.4,39.4,36.3,28.5,14.3,13.0 \mathrm{ppm}$. HRMS calcd for $\mathrm{C}_{14} \mathrm{H}_{19} \mathrm{~N}_{2} \mathrm{OS}^{+}: 263.1213$, found 263.1200 .

(4-(2-Isothiocyanatoethyl)phenyl)(morpholino)methanone (15). Yellow liquid, yield: $87 \%,{ }^{1} \mathrm{H}-\mathrm{NMR}(400 \mathrm{~Hz}$, $\left.\mathrm{CDCl}_{3}\right): \delta 7.38(\mathrm{~d}, 2 \mathrm{H}, J=8.0 \mathrm{~Hz}), 7.26(\mathrm{~d}, 2 \mathrm{H}, J=8.0 \mathrm{~Hz}), 3.73(\mathrm{t}, 2 \mathrm{H}, J=6.4 \mathrm{~Hz}), 3.44-3.76(\mathrm{~m}, 8 \mathrm{H}), 3.00$ $(\mathrm{t}, 2 \mathrm{H}, J=6.4 \mathrm{~Hz}) \mathrm{ppm} ;{ }^{13} \mathrm{C}-\mathrm{NMR}\left(100 \mathrm{~Hz}, \mathrm{CDCl}_{3}\right): \delta 170.2,139.1,134.5,129.1,127.8,67.0,60.4,46.2$, $36.3 \mathrm{ppm}$. MS (ESI, $\mathrm{m} / \mathrm{z}) 277.2\left(\mathrm{M}+\mathrm{H}^{+}\right)$. HRMS calcd for $\mathrm{C}_{14} \mathrm{H}_{16} \mathrm{~N}_{2} \mathrm{O}_{2} \mathrm{SNa}^{+}: 299.0825$, found 299.0832. (4-(2-Isothiocyanatoethyl)phenyl)(4-methylpiperazin-1-yl)methanone (16). Brown solid, yield: $80 \%,{ }^{1} \mathrm{H}-\mathrm{NMR}$ $\left(400 \mathrm{~Hz}, \mathrm{CDCl}_{3}\right): \delta 7.30-7.16(\mathrm{~m}, 4 \mathrm{H}), 3.70-3.38(\mathrm{~m}, 4 \mathrm{H}), 3.65(\mathrm{t}, 2 \mathrm{H}, J=6.8 \mathrm{~Hz}), 2.92(\mathrm{t}, 2 \mathrm{H}, J=6.8 \mathrm{~Hz})$, 2.41-2.36 (m, 4H), $2.24(\mathrm{~s}, 3 \mathrm{H}) ;{ }^{13} \mathrm{C}-\mathrm{NMR}\left(100 \mathrm{~Hz}, \mathrm{CDCl}_{3}\right): \delta 170.2,139.0,134.8,129.1,127.8,55.2,46.2$, 46.0, 36.4, $21.2 \mathrm{ppm}$; HRMS calcd for $\mathrm{C}_{15} \mathrm{H}_{20} \mathrm{~N}_{3} \mathrm{OS}^{+}$: 290.1322, found 290.1317.

4-(2-Isothiocyanatoethyl)-N-(pyridin-2-yl)benzamide (17). White solid, yield: $95 \%,{ }^{1} \mathrm{H}-\mathrm{NMR}(400 \mathrm{~Hz}$, $\left.\mathrm{CDCl}_{3}\right): \delta 8.66(\mathrm{~s}, 1 \mathrm{H}), 8.38(\mathrm{~d}, 1 \mathrm{H}, J=8.4 \mathrm{~Hz}), 8.29(\mathrm{~d}, 1 \mathrm{H}, J=4.0 \mathrm{~Hz}), 7.92(\mathrm{~d}, 2 \mathrm{H}, J=8.0 \mathrm{~Hz}), 7.74-7.78$ $(\mathrm{m}, 1 \mathrm{H}), 7.36(\mathrm{~d}, 2 \mathrm{H}, J=8.0 \mathrm{~Hz}), 7.06-7.09(\mathrm{~m}, 1 \mathrm{H}), 3.78(\mathrm{t}, 2 \mathrm{H}, J=7.2 \mathrm{~Hz}), 3.07(\mathrm{t}, 2 \mathrm{H}, J=7.2 \mathrm{~Hz}) \mathrm{ppm}$; ${ }^{13} \mathrm{C}-\mathrm{NMR}\left(100 \mathrm{~Hz}, \mathrm{CDCl}_{3}\right)$ : $\delta 151.6,148.1,141.8,138.7,133.4,129.5,127.9,120.1,114.3,46.1,36.5,29.8$ 
ppm; m.p. 139.6-141.3 ${ }^{\circ} \mathrm{C}$; MS (ESI, $\left.m / z\right) 284.1\left(\mathrm{M}+\mathrm{H}^{+}\right)$. HRMS calcd for $\mathrm{C}_{15} \mathrm{H}_{12} \mathrm{~N}_{3} \mathrm{OS}^{-}: 282.0707$, found 282.0697 .

4-(2-Isothiocyanatoethyl)-N-phenylbenzamide (18). White solid, yield: $91 \%,{ }^{1} \mathrm{H}-\mathrm{NMR}\left(400 \mathrm{~Hz}, \mathrm{CDCl}_{3}\right)$ : $\delta 7.85(\mathrm{~d}, 2 \mathrm{H}, J=8.0 \mathrm{~Hz}), 7.64(\mathrm{~d}, 2 \mathrm{H}, J=8.0 \mathrm{~Hz}), 7.39-7.33(\mathrm{~m}, 4 \mathrm{H}), 7.14-7.18(\mathrm{~m}, 1 \mathrm{H}), 3.77(\mathrm{t}, 2 \mathrm{H}$, $J=6.8 \mathrm{~Hz}), 3.06(\mathrm{t}, 2 \mathrm{H}, J=6.8 \mathrm{~Hz}) ;{ }^{13} \mathrm{C}-\mathrm{NMR}\left(100 \mathrm{~Hz}, \mathrm{CDCl}_{3}\right): \delta 165.5,141.3,138.0,134.1,129.4,129.2$, 127.7, 124.8, 120.4, 46.1, 36.4 ppm; m.p. $137.0-142.9^{\circ} \mathrm{C}$; HRMS calcd for $\mathrm{C}_{16} \mathrm{H}_{14} \mathrm{~N}_{2} \mathrm{OSNa}^{+}$: 305.0719 , found 305.0707 .

N-Benzyl-4-(2-isothiocyanatoethyl)benzamide (19). White solid, yield: $86 \%,{ }^{1} \mathrm{H}-\mathrm{NMR}\left(400 \mathrm{~Hz}, \mathrm{CDCl}_{3}\right)$ : $\delta 7.67(\mathrm{~d}, 2 \mathrm{H}, J=8.0 \mathrm{~Hz}), 7.25-7.16(\mathrm{~m}, 7 \mathrm{H}), 6.39(\mathrm{brs}, 1 \mathrm{H}), 4.54(\mathrm{~d}, 2 \mathrm{H}, J=5.6 \mathrm{~Hz}), 3.64(\mathrm{t}, 2 \mathrm{H}, J=6.8 \mathrm{~Hz})$, $2.92(\mathrm{t}, 2 \mathrm{H}, J=6.8 \mathrm{~Hz}) ;{ }^{13} \mathrm{C}-\mathrm{NMR}\left(100 \mathrm{~Hz}, \mathrm{CDCl}_{3}\right): \delta 167.1,140.9,138.3,133.5,129.2,128.9,128.0$, 127.8, 127.6, 46.1, 44.3, 36.4 ppm; m.p. $143.0-145.8{ }^{\circ} \mathrm{C}$; HRMS calcd for $\mathrm{C}_{17} \mathrm{H}_{16} \mathrm{~N}_{2} \mathrm{OSNa}^{+}$: 319.0876 , found 319.0877 .

4-(2-Isothiocyanatoethyl)-N-phenethylbenzamide (20). Yellow solid, yield: $97 \%,{ }^{1} \mathrm{H}-\mathrm{NMR}\left(400 \mathrm{~Hz}, \mathrm{CDCl}_{3}\right)$ : $\delta 7.58(\mathrm{~d}, 2 \mathrm{H}, J=8.0 \mathrm{~Hz}), 7.23(\mathrm{~d}, 2 \mathrm{H}, J=6.8 \mathrm{~Hz}), 7.18-7.14(\mathrm{~m}, 5 \mathrm{H}), 6.12(\mathrm{brs}, 1 \mathrm{H}), 3.66-3.60(\mathrm{~m}, 4 \mathrm{H})$, $2.92(\mathrm{t}, 2 \mathrm{H}, J=6.8 \mathrm{~Hz}), 2.85(\mathrm{t}, 2 \mathrm{H}, J=6.8 \mathrm{~Hz}) ;{ }^{13} \mathrm{C}-\mathrm{NMR}\left(100 \mathrm{~Hz}, \mathrm{CDCl}_{3}\right): \delta 167.2,140.8,139.0$, 133.8, 129.1, 128.9, 128.8, 127.5, 126.7, 46.1, 41.3, 36.4, 35.8 ppm; m.p. 98.2-102.5 ${ }^{\circ} \mathrm{C}$; HRMS calcd for $\mathrm{C}_{18} \mathrm{H}_{18} \mathrm{~N}_{2} \mathrm{OSNa}^{+}$: 333.1032, found 333.1046.

\subsection{Biological Cellular Assay}

3-(4,5-Dimethylthiazol-2-yl)-5-(3-carboxymethoxyphenyl)-2-(4-sulfophenyl)-2H-tetrazolium (MTS) powder and DMSO were bought from Sigma. DMEM medium, RPMI-1640 medium and fetal bovine serums were purchased from GIBCO.

\subsubsection{Cell Culture}

Human cancer cell lines Panc1 and Capan2 (pancreatic cancer), HGC27 (gastric cancer), and E6E7 were obtained from Sun-Yat Sen University Cancer Center. Cells were cultured in medium supplemented with $5-10 \%$ fetal bovine serum under the conditions of a humidified $5 \%$ carbon dioxide incubator. Every two to three days the medium was removed, when the confluence of cells reached about $80 \%$, and trypsin was used to digest the cells.

\subsubsection{MTS Assay}

Human cancer cells in logarithmic phase were inoculated in a 96-well plate (three wells for each drug concentration), with 1000-10,000 cells in each well. Once the cells were adherent, $100 \mu \mathrm{L}$ medium containing different concentrations of drugs were added, and after incubation for $72 \mathrm{~h}, 20 \mu \mathrm{L}$ MTS $(5 \mathrm{mg} / \mathrm{mL})$ was added, followed by incubation for another $4 \mathrm{~h}$. At the end of the incubation period, DMSO was used to dissolve purple formazen (the production of formazen was proportional to the number of viable cells). Then, the plate was shaken for 10-15 min and the optical density (OD) value of the plate was detected by microreader (Thermo Scientific, Waltham, USA) at a wavelength of $570 \mathrm{~nm}$. The survival rates were expressed as follows: survival rate $\%=$ mean OD value of drug treatment/mean OD value of control $\times 100 \%$ (Experiments were done for three times independently).

\subsubsection{Colony Formation}

Human cancer cells were seeded in 6-well plates at 500 cells per well. Then, cells were incubated with the indicated agents for 14 days, followed by fixation and staining with crystal violet. The samples were photographed and the colonies were counted. 


\subsubsection{Apoptosis and ROS Measurement}

Apoptosis was detected using Annexin V/PI staining. Cells were harvested and stained with Annexin V and PI for $15 \mathrm{~min}$, and apoptosis rates were analyzed using BD Calibur flow cytometry. Similarly, cells were stained for 30 min with DCF-DA for ROS detection. The samples were washed and analyzed using flow cytometry.

\subsubsection{GSH Levels}

Cellular GSH contents were measured using a GSH-Glo ${ }^{\mathrm{TM}}$ Glutathione Assay kit (Promega, WI, USA) according to the protocol provided by the manufacturer.

\section{Conclusions}

A concise preparation of isothiocyanates mediated by acetyl chloride and other electrophiles were studied. Using acetyl chloride, the expected isothiocyanates were obtained at good to excellent yields and the limitation of the often used tosyl chloride was overcome. These factors along its low cost would make acetyl chloride practical to prepare isothiocyanates. In our study, a series of novel isothiocyanates were designed and conveniently prepared using our newly discovered method. A biological evaluation on two malignant human cancer cell lines, pancreatic cancer Panc1 and gastric cancer HGC27, was investigated. Finally, compound $\mathbf{7}$ was found to have a better growth inhibition in the test cancer cells relative to PEITC that is one of the most studied naturally occuring isothiocyanates. In further study, compound 7 substantially inhibited the colony formation of pancreatic cancer cell lines Panc1 and Capan2, and caused better apoptosis than PEITC. Compound 7 also induced an increase in the levels of intracellular ROS and depleted the levels of GSH, which is one of the most recognized mechanisms of PEITC. Since several clinical trials with PEITC have been registered, our newly discovered compound 7 could provide therapeutic promise for the treatment of malignant tumors. Further study of the anticancer therapeutic effects of $\mathbf{7}$ is under way and will be reported in due time.

Supplementary Materials: Supplementary data including ${ }^{1} \mathrm{H}-\mathrm{NMR}$ spectrum and ${ }^{13} \mathrm{C}-\mathrm{NMR}$ spectrum can be available online.

Acknowledgments: We are grateful to the National Natural Science Foundation of China (81672952, 81430060), Guangdong Science and Technology Program (2014A030313196, 2013B051000034), Guangzhou Innovation Research Program (LCY201317), and Guangzhou Medicare Collaborative Innovation Program (201508020250).

Author Contributions: S.W., J.W. and B.L. conceived and designed the experiments; J.W., B.L. and X.L. performed the experiments; J.W. and B.L. analyzed the data; J.W., B.L., S.W. wrote the paper.

Conflicts of Interest: The authors declare no conflict of interest.

\section{References}

1. Ferguson, L.R.; Philpott, M. Cancer prevention by dietary bioactive components that target the immune response. Curr. Cancer Drug Targets 2007, 7, 459-464. [CrossRef] [PubMed]

2. Cheung, K.L.; Khor, T.O.; Huang, M.T.; Kong, A.N. Differential in vivo mechanism of chemoprevention of tumor formation in azoxymethane/dextran sodium sulfate mice by PEITC and DBM. Carcinogenesis 2010, 31, 880-885. [CrossRef] [PubMed]

3. Hu, R.; Kim, B.R.; Chen, C.; Hebbar, V.; Kong, A.N. The roles of JNK and apoptotic signaling pathways in PEITC-mediated responses in human HT-29 colon adenocarcinoma cells. Carcinogenesis 2003, 24, 1361-1367. [CrossRef] [PubMed]

4. Xiao, D.; Powolny, A.A.; Moura, M.B.; Kelley, E.E.; Bommareddy, A.; Kim, S.H.; Hahm, E.R.; Normolle, D.; Van Houten, B.; Singh, S.V. Phenethyl isothiocyanate inhibits oxidative phosphorylation to trigger reactive oxygen species-mediated death of human prostate cancer cells. J. Biol. Chem. 2010, 285, 26558-26569. [CrossRef] [PubMed] 
5. Runarsdottir, A.; Mannervik, B. A novel quasi-species of glutathione transferase with high activity towards naturally occurring isothiocyanates evolves from promiscuous low-activity variants. J. Mol. Biol. 2010, 401, 451-464. [CrossRef] [PubMed]

6. Syed Alwi, S.S.; Cavell, B.E.; Donlevy, A.; Packham, G. Differential induction of apoptosis in human breast cancer cell lines by phenethyl isothiocyanate, a glutathione depleting agent. Cell Stress Chaperones 2012, 17, 529-538. [CrossRef] [PubMed]

7. Yeomans, A.; Lemm, E.; Wilmore, S.; Cavell, B.E.; Valle-Argos, B.; Krysov, S.; Hidalgo, M.S.; Leonard, E.; Willis, A.E.; Forconi, F.; et al. PEITC-mediated inhibition of mRNA translation is associated with both inhibition of mTORC1 and increased eIF2alpha phosphorylation in established cell lines and primary human leukemia cells. Oncotarget 2016, 7, 74807-74819. [PubMed]

8. Cavell, B.E.; Syed Alwi, S.S.; Donlevy, A.M.; Proud, C.G.; Packham, G. Natural product-derived antitumor compound phenethyl isothiocyanate inhibits mTORC1 activity via TSC2. J. Nat. Prod. 2012, 75, 1051-1057. [CrossRef] [PubMed]

9. Wang, X.; Di Pasqua, A.J.; Govind, S.; McCracken, E.; Hong, C.; Mi, L.; Mao, Y.; Wu, J.Y.; Tomita, Y.; Woodrick, J.C.; et al. Selective depletion of mutant p53 by cancer chemopreventive isothiocyanates and their structure-activity relationships. J. Med. Chem. 2011, 54, 809-816. [CrossRef] [PubMed]

10. Chen, G.; Chen, Z.; Hu, Y.; Huang, P. Inhibition of mitochondrial respiration and rapid depletion of mitochondrial glutathione by beta-phenethyl isothiocyanate: mechanisms for anti-leukemia activity. Antioxid. Redox Signal. 2011, 15, 2911-2921. [CrossRef] [PubMed]

11. Trachootham, D.; Zhang, H.; Zhang, W.; Feng, L.; Du, M.; Zhou, Y.; Chen, Z.; Pelicano, H.; Plunkett, W.; Wierda, W.G.; et al. Effective elimination of fludarabine-resistant CLL cells by PEITC through a redox-mediated mechanism. Blood 2008, 112, 1912-1922. [CrossRef] [PubMed]

12. Trachootham, D.; Zhou, Y.; Zhang, H.; Demizu, Y.; Chen, Z.; Pelicano, H.; Chiao, P.J.; Achanta, G.; Arlinghaus, R.B.; Liu, J.; et al. Selective killing of oncogenically transformed cells through a ROS-mediated mechanism by beta-phenylethyl isothiocyanate. Cancer Cell 2006, 10, 241-252. [CrossRef] [PubMed]

13. Mays, J.R.; Weller Roska, R.L.; Sarfaraz, S.; Mukhtar, H.; Rajski, S.R. Identification, synthesis, and enzymology of non-natural glucosinolate chemopreventive candidates. ChemBioChem 2008, 9, 729-747. [CrossRef] [PubMed]

14. Vermeulen, M.; Zwanenburg, B.; Chittenden, G.J.; Verhagen, H. Synthesis of isothiocyanate-derived mercapturic acids. Eur. J. Med. Chem. 2003, 38, 729-737. [CrossRef]

15. Berglund, M.; Dalence-Guzman, M.F.; Skogvall, S.; Sterner, O. SAR studies of capsazepinoid bronchodilators 3: The thiourea part (coupling region) and the 2-(4-chlorophenyl)ethyl moiety (C-region). Bioorg. Med. Chem. 2008, 16, 2529-2540. [CrossRef] [PubMed]

16. Tsogoeva, S.B.; Hateley, M.J.; Yalalov, D.A.; Meindl, K.; Weckbecker, C.; Huthmacher, K. Thiourea-based non-nucleoside inhibitors of HIV reverse transcriptase as bifunctional organocatalysts in the asymmetric Strecker synthesis. Bioorg. Med. Chem. 2005, 13, 5680-5685. [CrossRef] [PubMed]

17. Isobe, T.; Ishikawa, T. 2-Chloro-1, 3-dimethylimidazolinium chloride. 2. Its application to the construction of heterocycles through dehydration reactions. J. Org. Chem. 1999, 64, 6989-6992. [CrossRef]

18. Munch, H.; Hansen, J.S.; Pittelkow, M.; Christensen, J.B.; Boas, U. A new efficient synthesis of isothiocyanates from amines using di-tert-butyl dicarbonate. Tetrahedron Lett. 2008, 49, 3117-3119. [CrossRef]

19. Wong, R.; Dolman, S.J. Isothiocyanates from tosyl chloride mediated decomposition of in situ generated dithiocarbamic acid salts. J. Org. Chem. 2007, 72, 3969-3971. [CrossRef] [PubMed]

20. Maisonneuve, P.; Lowenfels, A.B. Risk factors for pancreatic cancer: a summary review of meta-analytical studies. Int. J. Epidemiol. 2015, 44, 186-198. [CrossRef] [PubMed]

21. Von Hoff, D.D.; Ervin, T.; Arena, F.P.; Chiorean, E.G.; Infante, J.; Moore, M.; Seay, T.; Tjulandin, S.A.; Ma, W.W.; Saleh, M.N.; et al. Increased survival in pancreatic cancer with nab-paclitaxel plus gemcitabine. N. Engl. J. Med. 2013, 369, 1691-1703. [CrossRef] [PubMed]

22. Leung, W.K.; Wu, M.S.; Kakugawa, Y.; Kim, J.J.; Yeoh, K.G.; Goh, K.L.; Wu, K.C.; Wu, D.C.; Sollano, J.; Kachintorn, U.; et al. Screening for gastric cancer in Asia: Current evidence and practice. Lancet Oncol. 2008, 9, 279-287. [CrossRef]

23. Zhang, E.B.; Han, L.; Yin, D.D.; Kong, R.; De, W.; Chen, J. C-Myc-induced, long, noncoding H19 affects cell proliferation and predicts a poor prognosis in patients with gastric cancer. Med. Oncol. 2014, 31, 914. [CrossRef] [PubMed] 
24. Li, D.; Xie, K.; Wolff, R.; Abbruzzese, J.L. Pancreatic cancer. Lancet 2004, 363, 1049-1057. [CrossRef]

25. Raj, L.; Ide, T.; Gurkar, A.U.; Foley, M.; Schenone, M.; Li, X.; Tolliday, N.J.; Golub, T.R.; Carr, S.A.; Shamji, A.F.; et al. Selective killing of cancer cells by a small molecule targeting the stress response to ROS. Nature 2011, 475, 231-234. [CrossRef] [PubMed]

26. Trachootham, D.; Lu, W.; Ogasawara, M.A.; Nilsa, R.D.; Huang, P. Redox regulation of cell survival. Antioxid. Redox Signal. 2008, 10, 1343-1374. [CrossRef] [PubMed]

27. Franco, R.; Cidlowski, J.A. Apoptosis and glutathione: Beyond an antioxidant. Cell Death Differ. 2009, 16, 1303-1314. [CrossRef] [PubMed]

28. Chiang, Y.T.; Yen, Y.W.; Lo, C.L. Reactive oxygen species and glutathione dual redox-responsive micelles for selective cytotoxicity of cancer. Biomaterials 2015, 61, 150-161. [CrossRef] [PubMed]

Sample Availability: Samples of the compounds 6, 7 are available from the authors.

(C) 2017 by the authors. Licensee MDPI, Basel, Switzerland. This article is an open access article distributed under the terms and conditions of the Creative Commons Attribution (CC BY) license (http:/ / creativecommons.org/licenses/by/4.0/). 